Check for updates

Cite this: RSC Adv., 2017, 7, 26593

Received 22nd March 2017

Accepted 12th May 2017

DOI: $10.1039 / c 7 r a 03366 d$

rsc.li/rsc-advances

\section{PVDF/PVDF-g-PACMO blend hollow fiber membranes for hemodialysis: preparation, characterization, and performance $\uparrow$}

\author{
Zihan An, ${ }^{a}$ Rui Xu, ${ }^{a}$ Fengying Dai, ${ }^{a}$ Gaojian Xue, ${ }^{a}$ Xiaoling He, ${ }^{c}$ Yiping Zhao *a \\ and Li Chen (iD *ab
}

A novel approach to improve the biocompatibility of poly(vinylidene fluoride) (PVDF) hollow fiber membrane for hemodialysis is provided. A copolymer (PVDF-g-PACMO) containing the main chain of PVDF and amphiphilic side chains of polyacryloylmorpholine (PACMO) was blended with PVDF powder by a certain proportion and then was cast into a hollow fiber membrane via non-solvent induced phase separation (NIPS). The structure and performance of prepared membranes were characterized by $X$-ray photoelectron spectroscopy (XPS), field emission scanning electron microscopy (FE-SEM), mechanical testing, water contact angle measurement, multi-cycle filtration experiments, blood compatibility testing and dialysis simulation experiment. A higher weight ratio of PVDF-g-PACMO endows the blend membrane with larger membrane surface micro pores and a better hydrophilicity. Moreover, a high pure water flux and an improved blood compatibility with good dialysis property were obtained by enhancing weight ratio of PVDF-g-PACMO. Especially, when the weight ratio of PVDF-g-PACMO was 1/1 (i.e., 50/ 50), the water flux reached $200 \mathrm{~L} \mathrm{~m}^{-2} \mathrm{~h}^{-1}$ and the rejection ratio of BSA was more than $90 \%$, where the urea and lysozyme clearance rate was $80 \%$ and $62 \%$, respectively. All the results indicate that PVDF/ PVDF-g-PACMO blend hollow fiber membrane is promising to be applied in the field of hemodialysis.

\section{Introduction}

Renal function failure has become an increasing problem in the whole world, which may finally result in uremia. One of the vital cures for this disease is hemodialysis (HD). ${ }^{\mathbf{1 - 4}}$ Standard hemodialysis equipment consists of a dialysis machine, a water treatment system, dialysate and a dialyzer. The core component is the dialysis membrane through which mass transfer will be ongoing at both sides of blood and dialysate, i.e., excess metabolic wastes and toxins are removed while supplying calcium ion, bicarbonate ion and other substances. ${ }^{5-7}$ These membranes are mainly made of synthetic polymers materials such as polyether sulfone (PES) and polysulfone (PSF) due to their thermal and hydrolytic stability and good permeability of middlemolecular solute. ${ }^{8-11}$ However, the biocompatibility of these membranes material is still needed to be improved. ${ }^{\mathbf{1 2}}$ The adsorption and denaturation of proteins on the surface of PES

aState Key Laboratory of Separation Membranes and Membrane Processes, School of Material Science and Engineering, Tianjin Polytechnic University, Tianjin 300387, China. E-mail: tjpuchenlis@163.com

${ }^{b}$ School of Material Science and Engineering, Tianjin University of Technology, Tianjin 300384, China

'School of Environment and Chemical Engineering, Tianjin Polytechnic University, Tianjin 300387, China

$\dagger$ Electronic supplementary information (ESI) available. See DOI: 10.1039/c7ra03366d or PSF hollow fiber will further reduce biocompatibility of membranes ${ }^{13-16}$ and lead to clotting in the treatment. ${ }^{17}$ In addition, due to existing of isopropyl in their molecular structure, methyl radical is easy to be produced when contacting with strong oxidizer, and its residual will have an adverse influence on the human body. Therefore, finding a new polymer material with good biocompatibility to improve survival of patients and maintain high clearance of middle-molecular substance in comparison to conventional medical hemodialysis membrane materials is an urgent need.

PVDF is widely used in water treatment by means of its outstanding properties, such as chemical and thermal stability, good mechanical properties, and good membrane-forming properties. $^{\mathbf{1 8 - 2 1}}$ In recent years, PVDF membrane has been used in the medical field by virtue of its features, and much attention has been paid to improve its hydrophilicity and biocompatibility. Laroche et al. ${ }^{22}$ have confirmed that, from a biological point of view, PVDF sutures had excellent biocompatibility, especially for the minimal cell adsorption and tissue response. Zhu et al. ${ }^{23}$ indicated that, PVDF membrane is beneficial to increase solute rejection ratio in the process of bioseparation. Meng et al. $^{\mathbf{2 4}}$ grafted glycopolymer PGAMA onto PVDF membranes surface by ATRP, and as a result, the hydrophobicity and biocompatibility were improved dramatically. Idris et $a .^{25}$ blended multiwall carbon nanotube and PEG to PVDF dope solution, and proved that this modification 
improved the potential dialysis properties of PVDF membrane. However, the anticoagulant properties and dialysis performance of modified PVDF membranes used in hemodialysis field was scarcely reported.

Our previous work ${ }^{26,27}$ has come to the conclusion that the PVDF- $g$-PACMO copolymer membranes have a good resistance of protein adsorption and blood compatibility, and showed the potential using in terms of membrane contact blood. However, it is not suitable as a hemodialysis membrane due to structural defects of the flat membrane itself with respect to the hollow fiber membrane. Also, the membrane material anticoagulation has not been studied. Therefore, hollow membrane-forming technology is used in this paper to be more suitable for clinical application. In addition, PVP is added as the hydrophilizing additive for its excellent pore-forming effect and its good biocompatibility in biomedical field..$^{28,29}$ Moreover, hydrophilic PVP is also expected to show better affinity with PVDF- $g$-PACMO (hydrophilic polymer) to reduce its elution from the polymer membrane matrix during dialysis, ${ }^{29,30}$ and the PVP retained in the membrane structure provide the membrane with good biocompatibility. In this study, PVDF was blended with PVDF- $g$ PACMO copolymer and polyvinylpyrrolidone (PVP) in order to endow PVDF hollow membranes with good compatibility and dialysis performance. The blend hollow fiber membranes were prepared through the method of nonsolvent induced phase separation (NIPS). The influence of weight ratio of PVDF- $g$ PACMO to PVDF on the morphology, mechanical property, ultrafiltration performance, blood compatibility and simulated dialysis properties of PVDF/PVDF- $g$-PACMO blend hollow fiber membranes was further investigated.

\section{Materials and methods}

\subsection{Materials}

Poly(vinylidene fluoride) (PVDF, TA-1010, $153 \mathrm{kDa}$ ) was provided by Solvey Co. Ltd. (Brussels, Belgium) and was dried before use. $N$-Acryloylmorpholine (ACMO, 99\%) was purchased from Jiaxing Sicheng Chemical Co. Ltd. $N, N$-Dimethylformamide (DMF), poly(vinyl pyrrolidone) (PVP, K15) and Urea were supplied by Kemiou Chemical Co. Ltd. (Tianjin, China). AIBN ( $\geq 99 \%$ ) was provided by Shanghai Shisihewei Chemical Co. Ltd and was refined before use. Bovine serum albumin (BSA, 68 kDa) was supplied by Sigma Chemical Company. Lysozyme was provided by Shanghai Lanji biological Co. Ltd. The fresh rabbit blood ( sodium citrate/blood $=1 / 9$ ) was purchased from Tianjin Senrun Biotechnology Company. Other materials used as received were provided by commercial manufacturers.

\subsection{Preparation of PVDF- $g$-PACMO copolymer and the formation process of PVDF-g-PACMO/PVDF blend hollow fiber membranes}

The synthetic scheme of PVDF-g-PACMO copolymer and the formation process of membrane are shown in Fig. 1, which is described in detail in our previous study and results by FT-IR and NMR spectra have confirmed the copolymer were successfully synthesized. ${ }^{25}$ The membranes were prepared by the method of NIPS. ${ }^{31}$ In a typical procedure, $16 \mathrm{~g}$ blend polymers of PVDF/PVDF- $g$-PACMO, and PVP (8 g) were added into DMF (76 g) under stirring at $60{ }^{\circ} \mathrm{C}$ for $12 \mathrm{~h}$. After being filtered and degassed in the spinning kettle at $60{ }^{\circ} \mathrm{C}$, the spinning dope was spun through a spinneret with the dimensions of $0.4 \mathrm{~mm}$ (ID) and $0.8 \mathrm{~mm}$ (OD). The prepared membranes were firstly stored in water bath for $48 \mathrm{~h}$, and then they were frozen to dry until used. The PVDF/PVDF- $g$-PACMO blend hollow fiber membranes were designated as M-pristine, M-10, M-20, M-30, M-40, and M50 , respectively. In which the words/numbers in membrane ID presented the weight ratios of PVDF- $g$-PACMO relative to the total weight of PVDF and PVDF- $g$-PACMO.

\subsection{Characterization of PVDF-g-PACMO/PVDF blend hollow fiber membranes}

2.3.1 Surface chemistry and morphologies. The surface chemical compositions of blend hollow fiber membranes were detected by XPS (Geneis 60S, USA), where Al-K $\alpha$ was used as radiation resource $(1486.6 \mathrm{eV})$. The morphologies of the surface of the membranes were characterized by FESEM (Hitachi S4800 , Japan). In order to ensure the uniformity of the

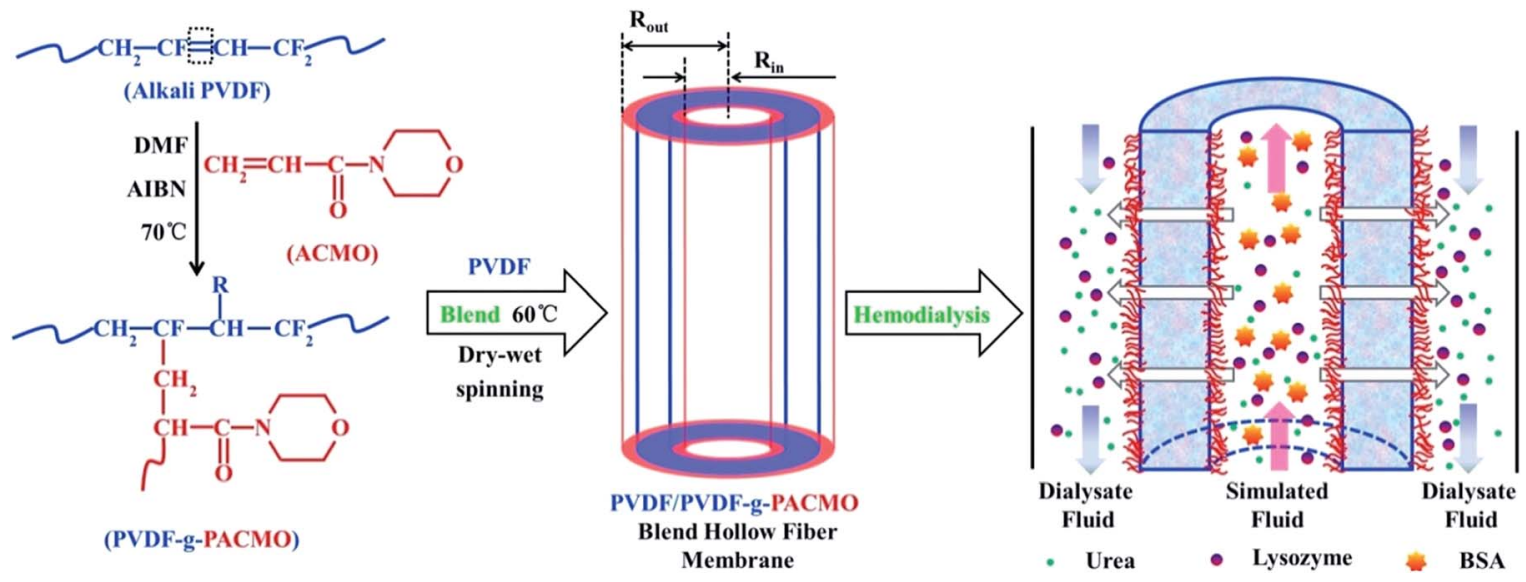

Fig. 1 Schematic diagram for the synthesis of PVDF-g-PACMO copolymer, membrane preparation and functionality of blend membranes. 
membrane, a high-resolution optical microscope (Olympus BX60M) was used to measure the diameters of each membrane.

2.3.2 Hydrophilicity and ultrafiltration experiments. The hydrophilicity of the membranes were characterized by water contact angle, and the measurement device was recorded with a contact angle meter (DSA 10, Germany). A piece of about $0.2 \times$ $2 \mathrm{~cm}^{2}$ membrane sample, obtained by cutting the blend hollow fiber membranes quickly with a surgical blade, was attached onto a glass slide. Then the water contact angle was recorded after the water dropped onto the surface of the membranes. Data were recorded as the average value of five measurements with standard deviation (SD).

Ultrafiltration experiments were tested through our homemade device as presented in Fig. S1. $\dagger$ The permeability of the blend hollow fiber membranes was evaluated by water permeation flux. The experiments were conducted at $25^{\circ} \mathrm{C}(0.1 \mathrm{MPa})$. The PWP flux was determined by:

$$
J=\frac{V}{\Delta t \times A}
$$

where $J$ represents the permeation flux $\left(\mathrm{L} \mathrm{m}^{-2} \mathrm{~h}^{-1}\right)$ for pure water, $V$ is the volume of permeate solution (L), $A$ is the membrane area $\left(\mathrm{m}^{2}\right)$ and $\Delta t$ is the permeation time $(\mathrm{h})$.

Solute rejection of membranes was characterized using BSA, lysozyme and urea solutions at a concentration of $1.0 \mathrm{~g} \mathrm{~L}^{-1}$. The rejection rate $(R)$ was calculated by:

$$
R=\left(1-\frac{C_{\mathrm{p}}}{C_{\mathrm{f}}}\right) \times 100 \%
$$

where $C_{\mathrm{p}}$ and $C_{\mathrm{f}}$ are concentrations of three solutions in permeate and feed solutions, respectively.

Multicycle water-BSA-water test was performed to test the long-term antifouling performance of blend membranes, the pure water flux $J_{\mathrm{w}}$, the flux of BSA solution $J_{\mathrm{B}}$ and the water flux after hydraulic cleaning $J_{\mathrm{r}}$ was recorded after each cycle, respectively. After the third cycle, the flux recovery ratio (FRR), total fouling index $\left(R_{\mathrm{t}}\right)$, reversible fouling index $\left(R_{\mathrm{r}}\right)$ and irreversible fouling index $\left(R_{\mathrm{ir}}\right)$ were calculated by:

$$
\begin{aligned}
\mathrm{FRR} & =\frac{J_{\mathrm{r}}}{J_{\mathrm{w}}} \times 100 \% \\
R_{\mathrm{t}} & =\frac{J_{\mathrm{w}}-J_{\mathrm{B}}}{J_{\mathrm{w}}} \\
R_{\mathrm{r}} & =\frac{J_{\mathrm{r}}-J_{\mathrm{B}}}{J_{\mathrm{w}}} \\
R_{\mathrm{ir}} & =\frac{J_{\mathrm{w}}-J_{\mathrm{r}}}{J_{\mathrm{w}}}
\end{aligned}
$$

2.3.3 Porosity and pore size of blend membranes. The porosity and pore size of the blend membranes was measured via capillary flow technology (CFT). Three pieces of fiber membranes with the length of $31 \mathrm{~mm}$ were fixed on the test component with $\mathrm{AB}$ glue. And then, the test component was installed on the instrument before testing. The membrane porosity, pore size distribution and mean pore size was recorded automatically.

\subsection{Blood compatibility}

2.4.1 Protein absorption. The protein adsorption tests were performed by BSA, and 3 pieces of fiber membrane samples were chosen $(30 \mathrm{~mm})$. Then they were incubated in BSA solution $\left(1.0 \mathrm{~g} \mathrm{~L}^{-1}\right)$ for $24 \mathrm{~h}$ until reaching adsorption equilibrium at room temperature. The protein absorption $(Q)$ was calculated by:

$$
Q=\frac{\left(C_{0}-C_{\mathrm{t}}\right) \times V}{S} \times 100 \%
$$

where $C_{0}$ and $C_{\mathrm{t}}$ are the BSA concentrations before and after adsorption, respectively; $V$ and $S$ are the volume of BSA solution and adsorption area of membrane sample, respectively; BSA concentrations were tested as our previous methods. ${ }^{26,27}$

2.4.2 Hemolysis ratio (HR). Membrane samples $(3 \times 30$ $\mathrm{mm}$ ) were rinsed with redistilled water and $0.9 \% \mathrm{NaCl}$ solutions (wt\%) for $10 \mathrm{~min}$, respectively, and then they were soaked in 10 $\mathrm{mL}$ of $\mathrm{NaCl}$ solutions $(0.9 \%)$ at $37^{\circ} \mathrm{C}$ for $30 \mathrm{~min}$. Afterwards, 200 $\mu \mathrm{L}$ fresh rabbit whole blood was added and was incubated for $1 \mathrm{~h}$. Finally, the blood was centrifuged at $2500 \mathrm{rpm}$ for $10 \mathrm{~min}$, and the absorbance of top clear layer was measured by UV-vis spectrophotometer $(545 \mathrm{~nm})$. Bidistilled water was used as positive control, while $\mathrm{NaCl}(0.9 \%)$ solution as negative control. The HR was calculated by eqn (8):

$$
\mathrm{HR}=\frac{A_{\mathrm{S}}-A_{\mathrm{N}}}{A_{\mathrm{P}}-A_{\mathrm{N}}} \times 100 \%
$$

where $A_{\mathrm{S}}, A_{\mathrm{P}}$, and $A_{\mathrm{N}}$ are the absorbance of the membrane samples, the positive control and the negative control, respectively.

2.4.3 Plasma coagulation. In order to characterize the anticoagulant activity of the blend membranes, clotting time such as activated partial thromboplastin time (APTT), prothrombin time (PT), and thrombin time (TT) were measured by an automatic blood coagulation analyzer (CA-600, Sysmex Corporation, Japan). The membrane sample $(3 \times 50 \mathrm{~mm})$ was immersed in PBS solution $(\mathrm{pH}=7.4)$ for $1 \mathrm{~h}$, then $3.0 \mathrm{~mL}$ fresh platelet-poor plasma (PPP), obtained by centrifuge at $4000 \mathrm{rpm}$ for $15 \mathrm{~min}$, was introduced and incubated at $37^{\circ} \mathrm{C}$ for $30 \mathrm{~min}$ after the PBS solution was removed. The test started when the PPP added to the test tube. Data were represented as average value $\pm \mathrm{SD}(n=3)$.

\subsection{Dialysis simulation experiment}

The simulated dialysis test cell was used to evaluate the property of prepared hemodialysis membranes and the schematic diagram is shown in Fig. 2. The dialysis test cell contained a membrane module, which consists of 10-20 fiber membranes. The solution containing urea $\left(1.5 \mathrm{~g} \mathrm{~L}^{-1}\right)$, lysozyme $\left(1.0 \mathrm{~g} \mathrm{~L}^{-1}\right)$ and BSA $\left(1.0 \mathrm{~g} \mathrm{~L}^{-1}\right)$ was used as simulated solution to simulate the real dialysis condition, ${ }^{32}$ and the bidistilled water was utilized dialysate solution. For a high-efficiency dialysis process as the paper reported, ${ }^{33}$ flow rate of simulation solution relative

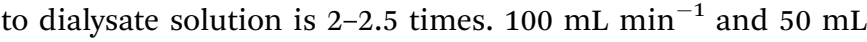
$\min ^{-1}$ were selected as the flow rates of simulation solution and dialysate, respectively. The operating temperature was kept at 


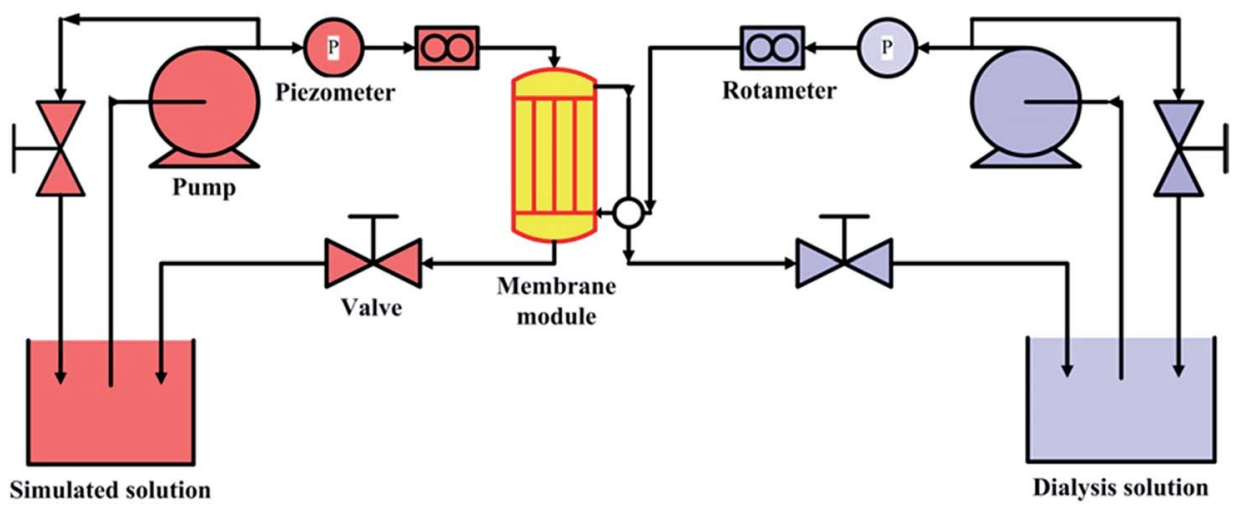

Fig. 2 Schematic diagram of simulated dialysis cell.

$37 \pm 0.5{ }^{\circ} \mathrm{C}$, and $2.0 \mathrm{~mL}$ of simulation solution outlet was collected after $4 \mathrm{~h}$. The clearance rate we used was the percentage of removed solute relative to original solute in blood simulation solution. Data were represented as average value \pm $\mathrm{SD}(n=3)$.

\section{Results and discussion}

\subsection{Surface chemistry}

The wide-scan XPS spectra and elemental content of blend hollow fiber membranes were shown in Fig. 3. In view of the figure, all the membranes had carbon (C), oxygen (O) and fluorine (F) peaks. Compared with pristine PVDF membrane (Mpristine), a new peak in $400 \mathrm{eV}$ attributed to nitrogen (N) signal in wide-scan XPS spectra of the blend hollow fiber membranes (M-10 to M-50). The content of $\mathrm{N}$ 1s increased with the increasing of the weight ratio, and the data were also shown in Fig. 3. With the increase of weight ratio of PVDF-g-PACMO, the $\mathrm{N}$ and $\mathrm{O}$ elemental percentage detected by XPS of the blend membranes increased from 0 (M-pristine) to $1.11 \%(\mathrm{M}-50)$ and from 4.39 (M-pristine) to $5.13 \%$ (M-50), respectively. All the results suggested that the hydrophilic segments of PACMO had segregation onto the membrane surface during the process of phase separation. And the amount of segregation increased with the increasing of weight ratio of PACMO copolymer.

\subsection{Surface morphology of blend membranes}

The surface morphologies of blend membranes were characterized by FE-SEM. In Fig. 4, the dense layer surface just as the classical surface structure of pure PVDF membrane could be observed on M-pristine, and a few of micropores appeared because of addition of PVP. In addition, pore sizes and porosity of blend membranes increased with the increasing weight ratio, which were consistent with the images of pore size distribution, and the results of pore structure parameters were shown in Table 1. The pore size data measured by CFT increases from 94 to $135 \mathrm{~nm}$ with increasing weight ratio of PVDF- $g$-PACMO copolymer, and the results were bigger than that shown in FESEM images. This problem was due to the reason that the pores in membranes measured by CFT were infiltrated the liquid metal mercury and the membrane pores were swelled, while dry membranes were used by SEM method. In addition, the increase of pore size and porosity had no influence on the mechanical properties, which was shown in Fig. S2.† a

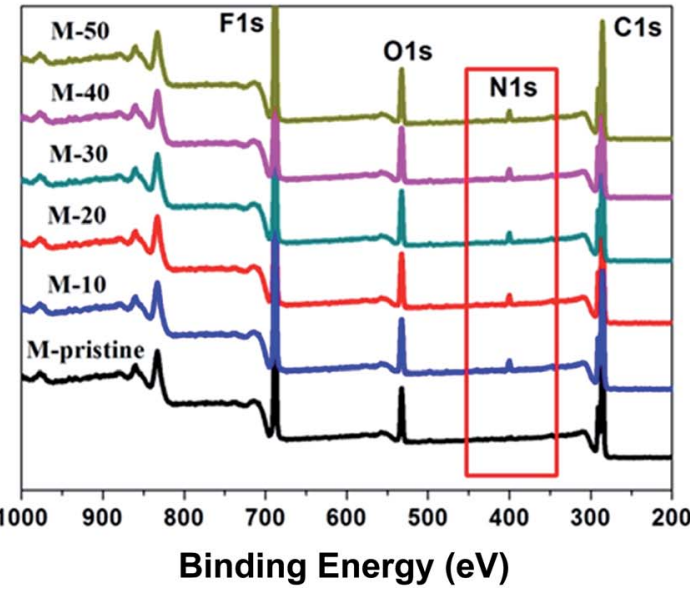

\begin{tabular}{cccccc}
\hline \multirow{2}{*}{ Sample } & \multicolumn{5}{c}{ Element (atom \%) } \\
\cline { 2 - 5 } & $\mathrm{C}$ & $\mathrm{N}$ & $\mathrm{O}$ & $\mathrm{F}$ & \\
\hline M-pristine & 51.68 & - & 4.39 & 43.92 & - \\
M-10 & 52.24 & 0.22 & 4.53 & 43.01 & 2.37 \\
M-20 & 52.81 & 0.45 & 4.67 & 42.10 & 3.11 \\
M-30 & 53.35 & 0.68 & 4.80 & 41.19 & 3.86 \\
M-40 & 53.91 & 0.90 & 4.95 & 40.28 & 5.08 \\
M-50 & 54.49 & 1.11 & 5.13 & 39.43 & 7.92 \\
\hline
\end{tabular}

Fig. 3 XPS wide-scan (a) and chemical compositions (b) of the surfaces of blend membranes. 

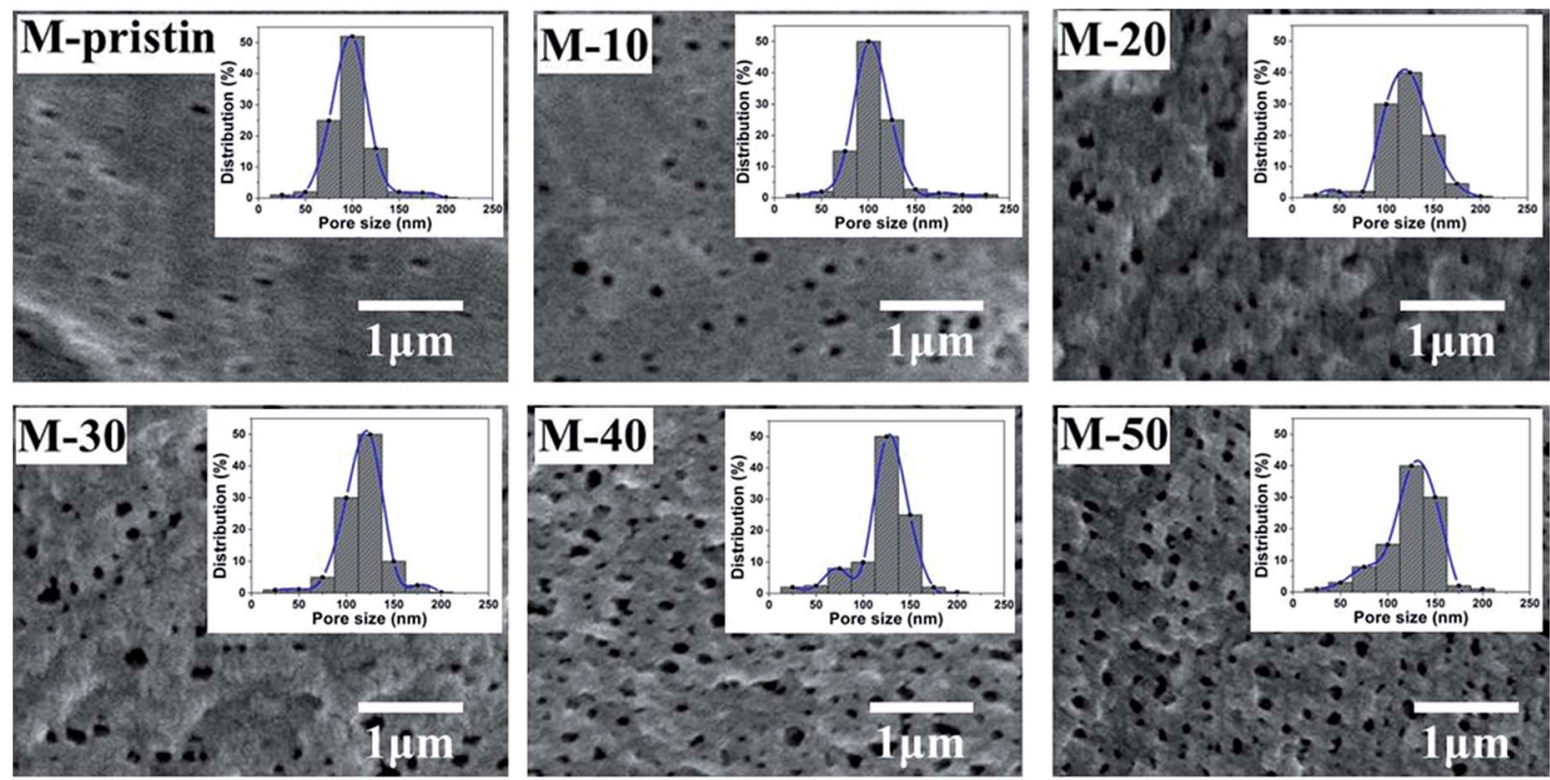

Fig. 4 SEM images and corresponding pore size distributions of blend membranes (the upper right corner of each figure corresponds to the membrane pore size distribution).

Table 1 Pore structure parameters of blend membranes (data inside and outside of the parentheses was the SEM test results and CFT results, respectively)

\begin{tabular}{lll}
\hline Membrane & Porosity (\%) & Mean pore size (nm) \\
\hline M-Pristine & $67.10(60.30)$ & $94(88)$ \\
M-10 & $72.50(65.40)$ & $105(95)$ \\
M-20 & $76.76(69.50)$ & $112(100)$ \\
M-30 & $79.46(71.20)$ & $120(105)$ \\
M-40 & $88.61(80.20)$ & $131(105)$ \\
M-50 & $89.22(82.60)$ & $135(108)$ \\
\hline
\end{tabular}

\subsection{Hydrophobicity of blend membranes}

When the materials contacted with blood, the equilibrium between hydrophilic and hydrophobic property of materials was very important. In this study, water contact angle is used to study the hydrophobicity of the membranes. As shown in Fig. 5, the initial contact angle of M-pristine was $100 \pm 0.5^{\circ}$. After being blended the PVDF- $g$-PACMO copolymer, the water contact angles of blend hollow fiber membranes decreased. When the weight ratio of PVDF- $g$-PACMO/PVDF is 10/90, the initial contact angle of the $\mathrm{M}-10$ was $78 \pm 0.4^{\circ}$, while the initial contact angle of the M-50 decreased to $62 \pm 0.7^{\circ}$. The result indicated that the surface of blend membranes became more hydrophilic after blending. In the process of the phase separation, a hydrophilic brush attributed to PACMO was formed on the membrane surface. Therefore, the hydrophilicity of membrane was improved. ${ }^{11}$ It can also be found that the contact angles of $\mathbf{M}$ pristine and M-10 had no obvious change with drop ages, while the contact angles of other membranes decreased rapidly. The fast decreasing contact angle for M-20 to M-50 membrane was mainly attributed to the high surficial hydrophilicity and more directly due to the hydrophilic nature of the PACMO side chains. As a result, water droplets could easily penetrate the surface of hydrophilic membranes with the drop age. And the result was in agreement with the increased pore size of blend hollow fiber membranes.

\subsection{Ultrafiltration properties}

In this study, time-dependent fluxes of water and BSA solutions were measured and the results were shown in Fig. 6. The increased pore sizes of the blend hollow fiber membranes were evaluated by water permeate flux and the rejection ratio of BSA. In addition, the antifouling properties were characterized by FRR and pollution index in the third cycle ultrafiltration. It can be seen that the water flux for M-pristine was $97 \mathrm{~L} \mathrm{~m}^{-2} \mathrm{~h}^{-1}$. The water flux of blend membranes was higher than that of the

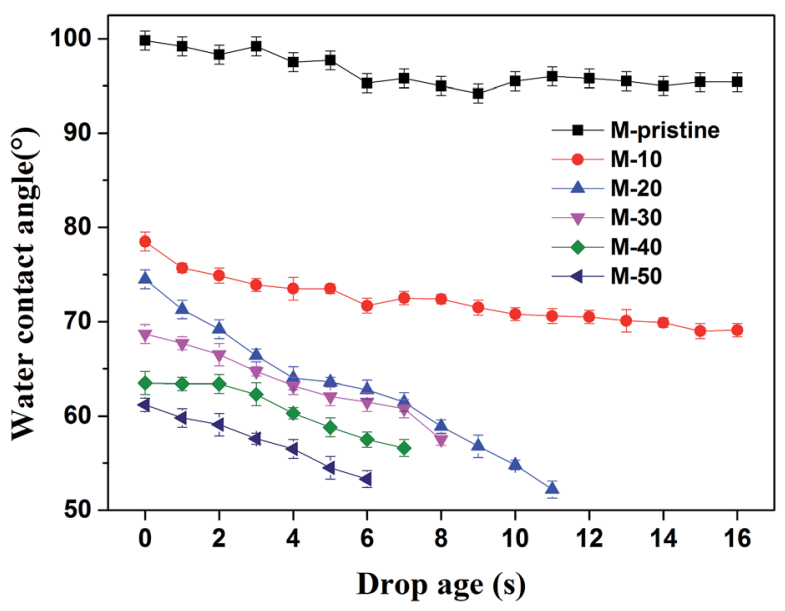

Fig. 5 Water contact angle of blend membranes. 

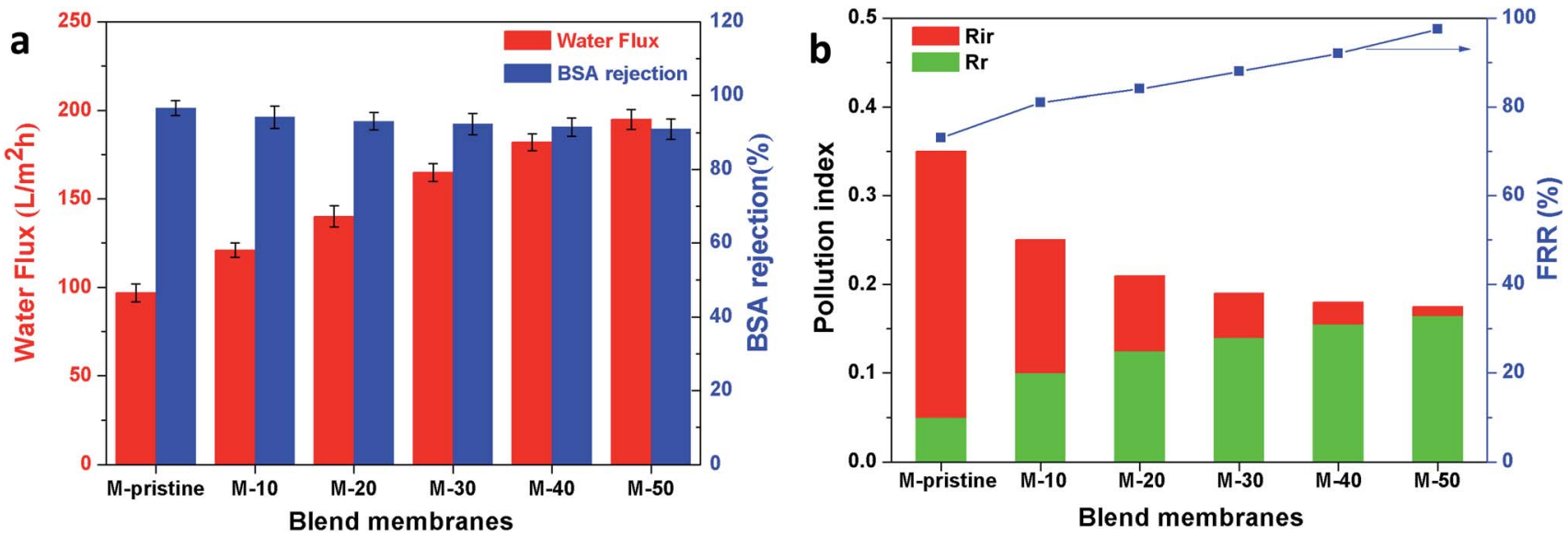

Fig. 6 (a) Pure water flux and BSA rejection ratios of blend membranes. (b) $R_{\mathrm{ir}}, R_{\mathrm{r}}$ and FRR of blend membranes in the third circulating filtration experiments $\left(R_{\mathrm{ir}}, R_{\mathrm{r}}\right.$ and FRR represented the irreversible fouling index, the reversible fouling index and the flux recovery ratio, respectively)

pristine PVDF membrane. Furthermore, the flux of M-50 increased to a certain degree, which can be up to $198 \mathrm{~L} \mathrm{~m}^{-2}$ $\mathrm{h}^{-1}$. The reason was the increasing pore diameter and pore number of blend hollow fiber membranes, which were consistent with the results of FE-SEM. Such water permeability was in conformity with the high-flux hemodialysis, which was beneficial to the clearance of middle-molecular toxin, and this water flux won't cause dehydration of patients. In addition, the flux recovery ratios of blend hollow fiber membranes increased with the increasing blend ratio, especially at the 3rd cycle of BSA/ water ultrafiltration, which increased from $72.5 \%$ to $98 \%$. Meanwhile, the fouling index decreased from 0.35 to 0.175 . These results indicated that all of the blend hollow fiber membranes, especially for M-40 and M-50, exhibited better antifouling property than M-pristine, which was attributed to the transfer of hydrophilic group of PACMO chain to blend membrane surface and then the hydration layer was formed.

\subsection{Blood compatibility}

3.5.1 Protein absorption and hemolysis ratio (HR). When blood is contacted with biomaterials, protein in the blood will be adsorbed on the surface of biomaterials. Moreover, protein adsorption has a great influence on hemodialysis, which can result in platelet adhesion and activation even blood coagulation. BSA adsorption amounts on the surface of the blend membranes were measured and the results were listed in

Table 2 BSA adsorption and hemolysis rate of blend membranes

\begin{tabular}{lll}
\hline & $\begin{array}{l}\text { BSA adsorption } \\
(\mu \mathrm{g}\end{array}$ & \\
Membrane & $\left.\mathrm{cm}^{-2}\right)$ & Hemolysis rate $(\%)$ \\
\hline M-Pristine & $120 \pm 0.65$ & $9.87 \pm 0.09$ \\
M-10 & $111 \pm 1.27$ & $7.74 \pm 0.21$ \\
M-20 & $106 \pm 0.89$ & $6.24 \pm 0.08$ \\
M-30 & $98 \pm 0.96$ & $5.01 \pm 0.06$ \\
M-40 & $82 \pm 0.81$ & $3.64 \pm 0.09$ \\
M-50 & $69 \pm 1.35$ & $1.94 \pm 0.16$
\end{tabular}

Table 2. The blend membranes exhibited lower protein adsorption compared with the pristine PVDF membrane. Especially for M-40 and M-50, the amount of protein adsorption decreased from $120 \mu \mathrm{g} \mathrm{cm} \mathrm{cm}^{-2}$ to $82 \mu \mathrm{g} \mathrm{cm} \mathrm{cm}^{-2}$ and $69 \mu \mathrm{g} \mathrm{cm}^{-2}$, respectively. The decreased protein adsorption might be attributed to the interactions of shielding electrostatic, van der Waals and hydration, which lead to a strong repulsive force between membrane surfaces with protein. ${ }^{34-37}$

Hemolysis ratio (HR) is also used to evaluate the blood compatibility, especially for the hematoclasis resulted from materials. As shown in Table 2, the HR value of M-40 and M-50 was $3.64 \%$ and $1.94 \%$, which were less than $5 \%$ and within safety levels for biomaterials. These results indicated that when the blend ratio of PVDF- $g$-PACMO copolymer was up to $40 \%$, the damage to erythrocyte could be weakened. As a result, the platelet adhesion and blood clotting was prevented.

3.5.2 Plasma coagulation. During clinical hemodialysis, clotting process after material contacting with the blood can be divided into activation of clotting factor, adhesion and aggregation of platelets, adhesion of red blood cell and activation of the complement system. Among them, plasma coagulation and thrombus are deadly for patients. In this study, PVDF was blended with PVDF- $g$-PACMO to enhance its anticoagulation. Activated partial thromboplastin time (APTT) was utilized to characterize blood coagulation from intrinsic pathway, and it can also be used in the detection of functional deficiencies. ${ }^{38,39}$ Prothrombin time (PT), adapted for its present principal uses in the screening, can be used to evaluate the extrinsic clotting pathway. ${ }^{38,39}$ Thrombin time (TT) is mainly used to characterize the anticoagulation ability of fibrinogen in plasma.9.40

Fig. 7 presented the APTT, PT and TT values of the blend membranes. It was observed that the APTTs, PTs and TTs of the blend membranes were prolonged compared with pristine PVDF membrane. Moreover, the PTs, APTTs and TTs increased with the increasing of the weight ratio. The M-50 showed the largest anticoagulant property with APTT value of $65.23 \mathrm{~s}$, which is nearly $62.5 \%$ higher than that of the pristine PVDF membrane. Furthermore, the APTT values of all the blend membranes increased compared with the pristine PVDF 


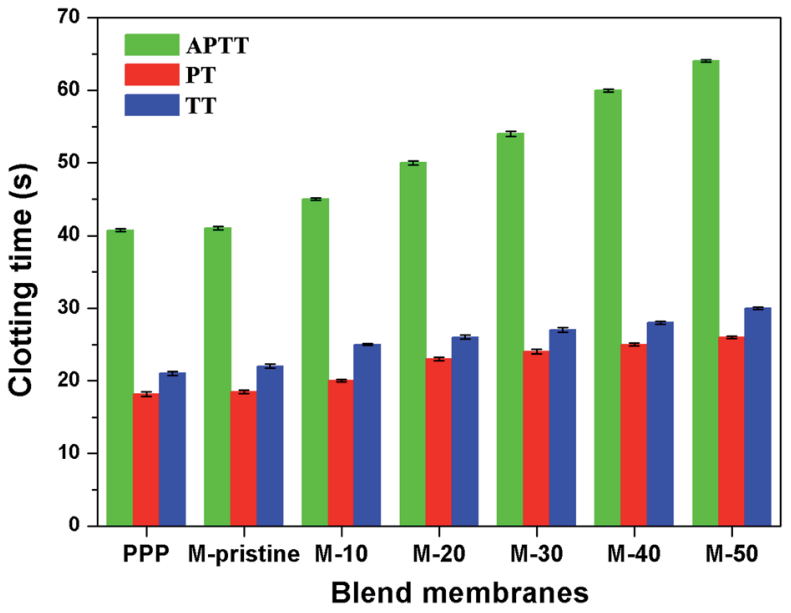

Fig. 7 APTTs, PTs and TTs for the PPP and the blend membranes (APTT, PT and TT represented the activated partial thromboplastin time, thrombin time and prothrombin time, respectively).

membrane, which indicated that the clotting process of PVDF membrane was mainly through intrinsic clotting pathway. However, the PT values and TT values of blend hollow fiber membranes were slightly higher than that of pristine PVDF membrane. The increase of clotting time was probably attributed to the hydrophilic brush from PACMO side chains and the main chain of PVP, which enriched the ether linkages and carbonyl groups at the interface between the blood and PVDF. Therefore, the introduction of PVDF- $g$-PACMO improved the anticoagulant ability and antifouling property of blend hollow membranes successfully.

\subsection{Dialysis performance}

During hemodialysis, urea and lysozyme are usually used to evaluate the dialysis performance of hemodialysis membrane. In the process of efficient hemodialysis, urea clearance rate should be more than $60 \%{ }^{41,42}$ During hemodialysis, middlemolecular proteins, namely $\beta_{2}$-microglobulin, may gather and finally harm the issues even cause dialysis-related amyloidosis. ${ }^{43}$ Because of composition consistency, lysozyme was used to evaluate the clearance rate of middle-molecular proteins instead of $\beta_{2}$-microglobulin. The clearance of urea and lysozyme and the retention of BSA were tested to characterize the dialysis performances of pristine PVDF membrane and blend membranes. Fig. 8(a) represents the rejection ratio of solutes with different molecular weight. BSA rejection ratio of all the membranes was more than $90 \%$, which was quite needed for preventing albumin from loss. As for lysozyme and urea, the ejection ratios are less than $40 \%$ and almost close to zero. As shown in Fig. 8(b), 82.5\% of urea has been cleaned for M-50 after simulating dialysis for $4 \mathrm{~h}$, which is higher than that of M-pristine (70\%) even the commercial parameters reported from Eknoyan. ${ }^{41}$ For the clearance rate of middle-molecule, $65 \%$ of lysozyme was cleaned out for M-50 and $40 \%$ for M-pristine after simulating dialysis for $4 \mathrm{~h}$ (Fig. 8 (b)). In summary, the increasing pore structure endowed the blend hollow fiber membranes with better dialysis property.
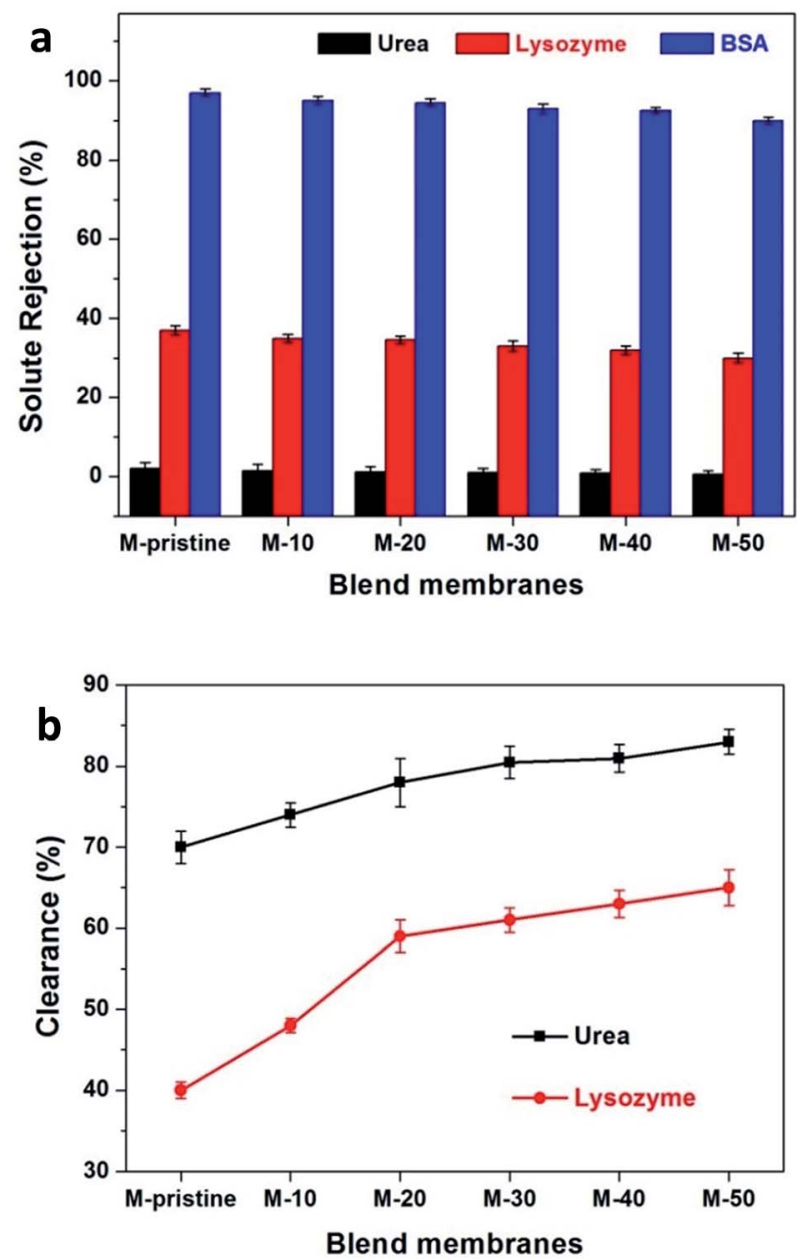

Fig. 8 (a) The solute rejection rate of PVDF and blend membranes were measured with urea, lysozyme and BSA. (b) Urea and lysozyme clearance of blend membranes.

\section{Conclusions}

PVDF blend hollow fiber membranes with good blood compatibility and dialysis performance, accomplished by blending PVDF with PVDF- $g$-PACMO, were developed successfully. The PVDF-g-PACMO was synthesized by free radical polymerization and directly blended with PVDF to prepare hollow fiber membranes through NIPS. Compared with the pristine PVDF membrane, the blend membranes exhibited better permeability performance and blood compatibility (lower BSA adsorption and prolonged whole blood clotting time) due to the modification by blending PVDF- $g$-PACMO. Furthermore, the dialysis performance of the blend membranes was improved with high clearance of urea and lysozyme, as well as high retention rate of BSA. These results indicated that the PVDF/ PVDF-g-PACMO blend hollow fiber membranes are potentially useful in the field of hemodialysis.

\section{Acknowledgements}

This work was financially sponsored by the National Natural Science Foundation of China (No. 21374078, 21174103 and 
51303129). Natural Science Foundation of Tianjin (15JCYBJC17900 and 14JCZDJC38300) and Program for Changjiang Scholars and Innovative Research Team in University of Ministry of Education of China (IRT13084). We would gratefully acknowledge the help of the Analytical and Testing Center at Tianjin Polytechnic University.

\section{References}

1 G. Xiong, X. Chen, X. Li, D. Fang, L. Zhang, L. Yang, L. Zhang, L. Yao, Z. He and L. Zhou, J. Formosan Med. Assoc., 2014, 113, 521-526.

2 T. V. Ariyaratne, Z. Ademi, S. J. Duffy, N. Andrianopoulos, B. Billah, A. L. Brennan, G. New, A. Black, A. E. Ajani, D. J. Clark, B. P. Yan, C.-H. Yap and C. M. Reid, Int. J. Cardiol., 2013, 168, 2783-2790.

3 H. Sugiyama, H. Yokoyama, H. Sato, T. Saito, Y. Kohda, S. Nishi, K. Tsuruya, H. Kiyomoto, H. Iida, T. Sasaki, M. Higuchi, M. Hattori, K. Oka, S. Kagami, T. Kawamura, T. Takeda, H. Hataya, Y. Fukasawa, A. Fukatsu, K. Morozumi, N. Yoshikawa, A. Shimizu, H. Kitamura, Y. Yuzawa, S. Matsuo, Y. Kiyohara, K. Joh, M. Nagata, T. Taguchi and H. Makino, Clin. Exp. Nephrol., 2013, 17, 155-173.

4 C. Ahn, T. Y. Koo, J. C. Jeong, M. Kim, J. Yang, J. Lee, S. I. Min, J. E. Lee, M. S. Kim, O. J. Kwon, S. J. Kim, Y. H. Kim, Y. H. Kim, B. S. Choi, S. J. N. Choi, D. H. Lee, S. Y. Chung, W. H. Cho and Y. S. Kim, Transplant. Proc., 2014, 46, 425-430.

5 N. C. V. Melo, R. M. A. Moyses, R. M. Elias and M. C. M. Castro, Hemodialysis International, 2014, 18, 473-480.

6 Z. Y. Oo, R. Deng, M. Hu, M. Ni, K. Kandasamy, M. S. Bin Ibrahim, J. Y. Ying and D. Zink, Biomaterials, 2011, 32, 8806-8815.

7 G. J. Dahe, R. S. Teotia, S. S. Kadam and J. R. Bellare, Biomaterials, 2011, 32, 352-365.

8 S. Nie, J. Xue, Y. Lu, Y. Liu, D. Wang, S. Sun, F. Ran and C. Zhao, Colloids Surf., B, 2012, 100, 116-125.

9 L. Li, C. Cheng, T. Xiang, M. Tang, W. Zhao, S. Sun and C. Zhao, J. Membr. Sci., 2012, 405-406, 261-274.

10 J. Y. Park, M. H. Acar, A. Akthakul, W. Kuhlman and A. M. Mayes, Biomaterials, 2006, 27, 856-865.

11 F. Ran, S. Nie, W. Zhao, J. Li, B. Su, S. Sun and C. Zhao, Acta Biomater., 2011, 7, 3370-3381.

12 V. Sirolli, E. Ballone, S. Di Stante, L. Amoroso and M. Bonomini, Int. J. Artif. Organs, 2002, 25, 529-537.

13 D. Basmadjian, M. V. Sefton and S. A. Baldwin, Biomaterials, 1997, 18, 1511-1522.

14 A. Fujimori, H. Naito and T. Miyazaki, Artif. Organs, 1998, 22, 1014-1017.

15 G. Yin, J.-C. Janson and Z. Liu, J. Membr. Sci., 2000, 178, 99105.

16 H. R. Roberts, Kidney Blood Pressure Res., 2012, 35, 299-394. 17 M. Irfan and A. Idris, Mater. Sci. Eng., C, 2015, 56, 574-592.
18 K. Tu, P. Shen, J. Li, B. Fan, C. Yang and R. Du, J. Appl. Polym. Sci., 2015, 132, 1-6.

19 F. Liu, N. A. Hashim, Y. Liu, M. R. M. Abed and K. Li, J. Membr. Sci., 2011, 375, 1-27.

20 H. P. Srivastava, G. Arthanareeswaran, N. Anantharaman and V. M. Starov, Desalination, 2011, 282, 87-94.

21 M. Khayet, Adv. Colloid Interface Sci., 2011, 164, 56-88.

22 G. Laroche, Y. Marois, R. Guidoin, M. W. King, L. Martin, T. How and Y. Douville, J. Biomed. Mater. Res., 1995, 29, 1525-1536.

23 L.-P. Zhu, J.-Z. Yu, Y.-Y. Xu, Z.-Y. Xi and B.-K. Zhu, Colloids Surf., B, 2009, 69, 152-155.

24 J. Yuan, J.-Q. Meng, Y.-L. Kang, Q.-Y. Du and Y.-F. Zhang, Appl. Surf. Sci., 2012, 258, 2856-2863.

25 K. H. Chan, E. T. Wong, M. I. Khan, A. Idris and N. M. Yusof, J. Ind. Eng. Chem., 2014, 20, 3744-3753.

26 J. Liu, X. Shen, Y. Zhao and L. Chen, Ind. Eng. Chem. Res., 2013, 52, 18392-18400.

27 X. Shen, J. Liu, X. Feng, Y. Zhao and L. Chen, J. Biomed. Mater. Res., Part A, 2015, 103, 683-692.

28 M. Irfan, A. Idris, N. M. Yusof, N. F. M. Khairuddin and H. Akhmal, J. Membr. Sci., 2014, 467, 73-84.

29 Q. Yang, T.-S. Chung and M. Weber, J. Membr. Sci., 2009, 326, 322-331.

30 M. Sun, Y. Su, C. Mu and Z. Jiang, Ind. Eng. Chem. Res., 2010, 49, 790-796.

31 D.-M. Wang and J.-Y. Lai, Curr. Opin. Chem. Eng., 2013, 2, 229-237.

32 X. Yu, L. Shen, Y. Zhu, X. Li, Y. Yang, X. Wang, M. Zhu and B. S. Hsiao, J. Membr. Sci., 2017, 523, 173-184.

33 G. D. Graves, Nephrology Nursing Journal, 2001, 28, 23-30.

34 L. Zou, I. Vidalis, D. Steele, A. Michelmore, S. P. Low and J. Q. J. C. Verberk, J. Membr. Sci., 2011, 369, 420-428.

35 W. Gu, Q. Li, H. Lu, L. Fang, Q. Chen, Y. Yang and H. Gao, Chem. Commun., 2015, 51, 4715-4718.

36 H. Han, D. Liu, H. Lu, W. Gu and H. Gao, RSC Adv., 2014, 4, 40882-40891.

37 L. Wang, Y. Yang, M. Zhu, G. Qiu, G. Wu and H. Gao, RSC Adv., 2014, 4, 6478-6485.

38 M. M. Flanders, R. Crist and G. M. Rodgers, Clin. Chem., 2003, 49, 169-172.

39 N. Key, M. Makris, D. O'Shaughnessy and D. Lillicrap, Practical Hemostasis and Thrombosis, Wiley-Blackwell, 2009. 40 R. T. S. Jim, J. Lab. Clin. Med., 1957, 50, 45-60.

41 G. Eknoyan, G. J. Beck, A. K. Cheung, J. T. Daugirdas, T. Greene, J. W. Kusek, M. Allon, J. Bailey, J. A. Delmez, T. A. Depner, J. T. Dwyer, A. S. Levey, N. W. Levin, E. Milford, D. B. Ornt, M. V. Rocco, G. Schulman, S. J. Schwab, B. P. Teehan and R. Toto, N. Engl. J. Med., 2002, 347, 2010-2019.

42 G. Lesaffer, R. De Smet, N. Lameire, A. Dhondt, P. Duym and R. Vanholder, Nephrol., Dial., Transplant., 2000, 15, 50-57. 43 L. M. Dember, J. Am. Soc. Nephrol., 2006, 17, 3458-3471. 